\title{
A Study for Improving Accuracy of Overhead Con- ductor Temperature Model under Large Discrete Steps
}

\author{
Xiong Chen ${ }^{1}$, Renzhi $\mathrm{Hu}^{1}$, Yujuan Hong ${ }^{2, *}$ and Zhanfeng Ying ${ }^{2}$ \\ ${ }^{1}$ NARI Technology Co., Ltd, Jiangsu Nanjing, China, 211000 \\ ${ }^{2}$ School of Energy and Power Engineering, Nanjing University of Science and Technology, Jiangsu Nanjing, China, 210094 \\ ${ }^{*}$ Corresponding author
}

\begin{abstract}
The accuracy of overhead conductor temperature model is easily affected by the sampling frequency of current and weather conditions. Due to the cost limitation and communication difficulty of the monitoring systems, the current and weather conditions is commonly sampled with the large sampling steps in the real engineering. The accuracy of calculated conductor temperature would be decreased, since the overhead conductor temperature model have to be calculated with the large discrete steps. In this paper, a method, combining the fourth-order RungeKutta algorithm and quadratic function interpolation, is proposed to improve the accuracy of overhead conductor temperature model under the large discrete steps. The calculating example indicated the validity of proposed method.
\end{abstract}

Keywords-conductor temperature; quadratic function interpolation; calculation precision

\section{INTRODUCTION}

Since the conductor temperature reflects the dynamic thermal performance of overhead transmission lines, it is an important parameter for the operation safety assessment of transmission line. In the recent years, the conductor temperature becomes more and more important for the power system, because it is widely used in the researches such as transmission line life cycle assessment ${ }^{[1]}$, local hotspot location ${ }^{[2]}$, line dynamic rating estimation ${ }^{[3-6]}$ and electro-thermal coordination ${ }^{[7]}$ theory development.

At present, a conductor temperature model, expressed as a first-order nonlinear differential equation, is widely used to calculate conductor temperature. This model comes from IEEE standard 738-2012 $2^{[8]}$ and can be called IEEE model for short. In IEEE model, the conductor temperature is mainly affected by the current and weather conditions so that the accuracy of this model is affected by the time resolution of current and weather conditions. Although in the IEEE standard, the forward Euler algorithm is recommended to discretize IEEE model, this algorithm would lead to large calculation error when the current and weather conditions are sampled with the large steps. In the actual project, the sampling frequency of the line monitoring system is hard to improve dramatically under the constraints of power supply mode, communication mode, maintenance difficulty and system cost ${ }^{[9-10]}$. That means it is necessary to improve the accuracy of conductor temperature model under the large discrete steps.
In this paper, a method combining the fourth-order RungeKutta algorithm and quadratic function interpolation, is proposed to calculate conductor temperature model. The calculating example indicates that the proposed method can improve calculated accuracy even though the model is discretized with the large discrete steps.

\section{Overhead Conductor Temperature Model}

The IEEE standard shows that the overhead conductor temperature is mainly affected by current and ambient weather conditions. Therefore, the overhead conductor temperature can be expressed as:

$$
q_{c}+q_{r}+C \cdot \frac{\mathrm{d} T_{c}}{\mathrm{~d} t}=q_{s}+I^{2} \cdot R\left(T_{c}\right)
$$

where $q_{c}$ is convection heat loss rate, $q_{r}$ is radiated heat loss rate, $C$ is specific heat of conductor material, $T_{c}$ is conductor temperature, $q_{s}$ is heat gain rate from sun, $I$ is conductor current, $R\left(T_{c}\right)$ is AC resistance of conductor at temperature $T_{c}$.

The parameters in (1) can be calculated by:

$$
\begin{aligned}
& \int K_{\text {angle }}\left[1.01+1.35\left(\frac{D_{o} \rho_{f} V_{w}}{\mu_{f}}\right)^{0.52}\right] k_{f}\left(T_{c}-T_{a}\right) \\
& \text { for low wind speed } \\
& q_{c}=\left\{0.754 K_{\text {angle }}\left(\frac{D_{o} \rho_{f} V_{w}}{\mu_{f}}\right)^{0.6} k_{f}\left(T_{c}-T_{a}\right)\right. \\
& \begin{array}{r}
\text { for high wind speed } \\
3.645 \rho_{f}^{0.5} D_{o}^{0.75}\left(T_{c}-T_{a}\right)^{1.25} \text { for nature convection }
\end{array}
\end{aligned}
$$




$$
\begin{gathered}
q_{r}=17.8 D_{o} \varepsilon\left[\left(\frac{T_{c}+273}{100}\right)^{4}-\left(\frac{T_{a}+273}{100}\right)^{4}\right] \\
C=m C_{p} \\
q_{s}=\alpha K_{\text {solar }} Q_{s} \sin (\theta) A^{\prime}
\end{gathered}
$$

Where $K_{\text {angle }}$ is wind direction factor, $D_{o}$ is outside diameter of conductor, $\rho_{f}$ is density of air, $V_{w}$ is speed of air stream at conductor, $\mu_{f}$ is absolute viscosity of air, $k_{f}$ is thermal conductivity of air, $T_{a}$ is ambient air temperature, $\varepsilon$ is emissivity, $m C_{p}$ is total heat capacity of conductor, $\alpha$ is solar absorptivity, $K_{\text {solar }}$ is solar altitude correction factor, $Q_{s}$ is total solar and sky radiated heat intensity, $\theta$ is effective angle of incidence of the sunshine rays, $A$ is projected area of conductor.

Obviously, this overhead conductor temperature model is a first-order nonlinear differential equation. Although the forward Euler algorithm is recommended to discretize IEEE model with 1-min time step in IEEE standard, this algorithm would lead to large calculation error when the current and weather conditions are sampled with the time steps larger than 1-min.

In fact, the Runge-Kutta algorithm is regionalized as a high accuracy discretization algorithm under the large discrete step. In numerical analysis, the Runge-Kutta algorithms are a family of implicit and explicit iterative methods. The most widely known member of the Runge-Kutta family is generally referred to as "fourth-order Runge-Kutta" or "RK4" and $s_{1}, s_{2}, \mathrm{~L}, s_{m}$ it can be expressed as:

$$
T_{c}^{i}=T_{c}^{i-1}+\frac{h}{6}\left(s_{1}+2 s_{2}+2 s_{3}+s_{4}\right)
$$

Where $s_{1}, s_{2}, s_{3}$ and $s_{4}$ are the slope term, and they can be calculated as:

$$
\left\{\begin{array}{l}
s_{1}=\frac{1}{m C_{p}}\left[R\left(T_{c}^{i-1}\right) I^{2}+q_{s}-q_{c}\left(T_{c}^{i-1}\right)-q_{r}\left(T_{c}^{i-1}\right)\right] \\
s_{2}=\frac{1}{m C_{p}}\left[R\left(T_{c}^{i-1}+\frac{h s_{1}}{2}\right) I^{2}+q_{s}-q_{c}\left(T_{c}^{i-1}+\frac{h s_{1}}{2}\right)-q_{r}\left(T_{c}^{i-1}+\frac{h s_{1}}{2}\right)\right] \\
s_{3}=\frac{1}{m C_{p}}\left[R\left(T_{c}^{i-1}+\frac{h s_{2}}{2}\right) I^{2}+q_{s}-q_{c}\left(T_{c}^{i-1}+\frac{h s_{2}}{2}\right)-q_{r}\left(T_{c}^{i-1}+\frac{h s_{2}}{2}\right)\right] \\
s_{4}=\frac{1}{m C_{p}}\left[R\left(T_{c}^{i-1}+h s_{3}\right) I^{2}+q_{s}-q_{c}\left(T_{c}^{i-1}+h s_{3}\right)-q_{r}\left(T_{c}^{i-1}+h s_{3}\right)\right]
\end{array}\right.
$$

Compared with the forward Euler algorithm, the fourth- order Runge-Kutta algorithm have higher accuracy because the weighted slope $\left(s_{1}+2 s_{2}+2 s_{3}+s_{4}\right) / 6$ is adopted to calculate the conductor temperature at next time point and this weighted slope can estimate the slope between the time interval with higher accuracy.

\section{THE ACCURACY ANALYSIS OF OVERHEAD CONDUCTOR TEMPERATURE MODEL UNDER THE LARGE DISCRETE STEPS}

In order to analyze the accuracy of overhead conductor temperature model under the large discrete step, a calculating example is performed. In this calculating example, the conductor type is LGJ240/30 and its thermal and electrical parameters are shown in Table I.

TABLE I. THE THERMAL PARAMETERS OF THE CONDUCTOR

\begin{tabular}{|c|c|c|c|c|}
\hline $\boldsymbol{C}_{\boldsymbol{p}}\left(\mathbf{J} / \boldsymbol{m} \cdot{ }^{\circ} \boldsymbol{C}\right)$ & $\boldsymbol{D}_{\boldsymbol{o}}(\boldsymbol{m})$ & $\boldsymbol{R}_{\boldsymbol{a}}\left(\mathbf{\Omega} / \boldsymbol{m}, \mathbf{2 0}{ }^{\circ} \boldsymbol{C}\right)$ & $\boldsymbol{T}_{\boldsymbol{a}}\left({ }^{\circ} \boldsymbol{C}\right)$ & $a$ \\
\hline 852.72 & 21.6 & 0.1181 & 20 & 0.00386 \\
\hline
\end{tabular}

Moreover, the weather conditions and current are assumed to change in real-time during the conductor temperature calculation. The variation of wind speed, sun intensity and current are shown in Figure I to Figure III respectively.

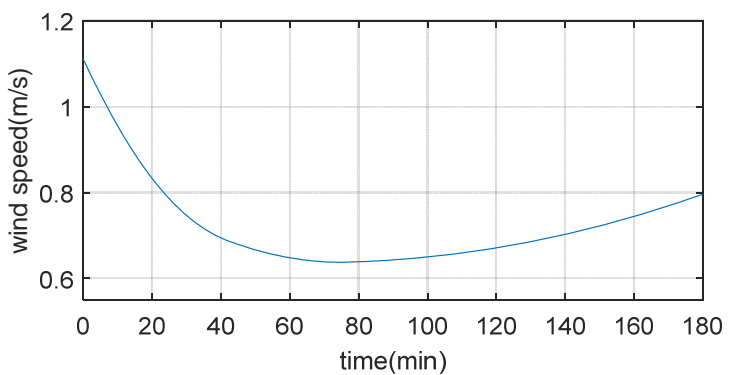

FIGURE I. VARIATION OF THE WIND SPEED

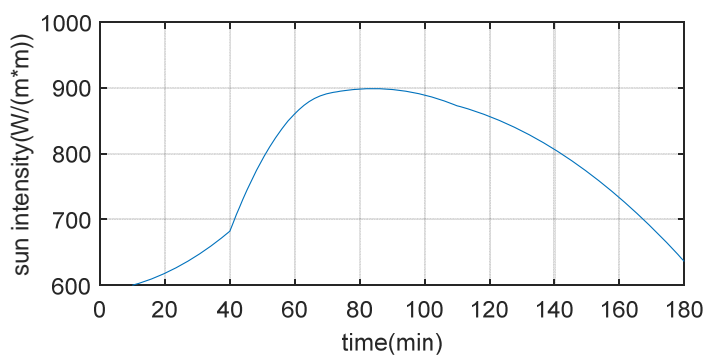

FIGURE II. VARIATION OF THE SUN INTENSITY

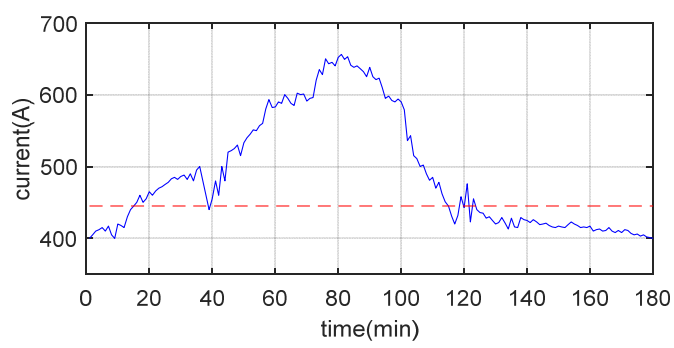

FIGURE III. VARIATION OF THE LINE CURRENT 
Then, the conductor temperature is calculated with the discrete steps of $1 \mathrm{~min}, 5 \mathrm{~min}, 10 \mathrm{~min}$ and $20 \mathrm{~min}$. Figure IV and Figure $\mathrm{V}$ show the calculated conductor temperature and the calculated error respectively. According to the IEEE standard, the conductor temperature, calculated with the discrete step of $1 \mathrm{~min}$, can be treated as results with the highest accuracy. Hence, it can be found that the calculated accuracy is decreased with the increasing of the discrete step. Especially, the maximum conductor temperature $\left(71.73^{\circ} \mathrm{C}\right)$ cannot be calculated when the discrete step is set to be $20 \mathrm{~min}$. This is because the fourthorder Runge-Kutta algorithm miss the time point corresponding to the maximum conductor temperature in such large discrete step. This means the result calculated with the fourth-order Runge-Kutta algorithm would be unreliable under the discrete steps, although the fourth-order Runge-Kutta algorithm have higher accuracy than the forward Euler algorithm.

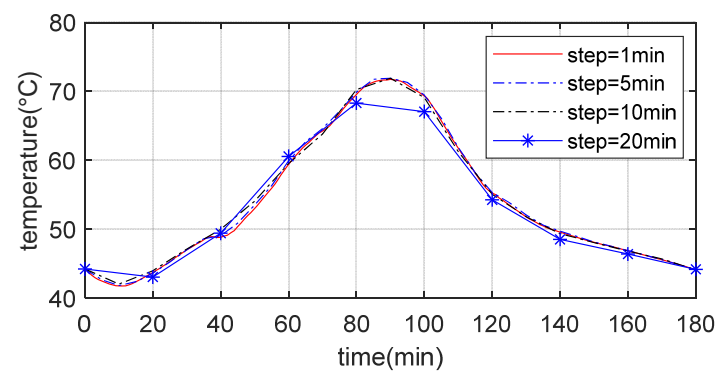

FIGURE IV. THE TEMPERATURE OF LINE

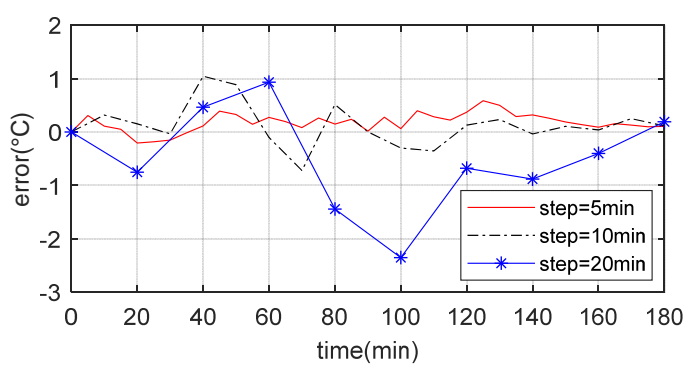

FIGURE V. THE ERROR OF LINE TEMPERATURE

\section{The PROPOSED METHOD}

\section{A. Principle of Proposed Method}

As mentioned above, the fourth-order Runge-Kutta algorithm would miss the maximum conductor temperature under the large discrete steps. To improve the accuracy of overhead conductor temperature model, a method combining the fourthorder Runge-Kutta algorithm and quadratic function interpolation, is proposed in this paper.

The proposed method calculates conductor temperature by the fourth-order Runge-Kutta arithmetic in the first step. Then, the time points $i$ corresponding to the monotonicity change of conductor temperature are found out according to the calculated results. After that, the current and weather conditions during the time point $i-1$ to $i+1$, can be supplemented by the quadratic interpolation. The quadratic interpolation function can be expressed as:

$$
\left\{\begin{array}{l}
v_{t}=a_{v} t^{2}+b_{v} t+c_{v} \\
S_{t}=a_{s} t^{2}+b_{s} t+c_{s} \\
I_{t}=a_{I} t^{2}+b_{I} t+c_{I}
\end{array}\right.
$$

Where $v_{t}, S_{t}$ and $I_{t}$ are supplemented wind speed, sun intensity and line current, respectively. The coefficients in the quadratic interpolation function can be calculated as (take the coefficients $a_{v}, b_{v}$ and $c_{v}$ as example):

$$
\left\{\begin{array}{l}
v_{i-1}=a_{v} t_{i-1}^{2}+b_{v} t_{i-1}+c_{v} \\
v_{i}=a_{v} t_{i}^{2}+b_{v} t_{i}+c_{v} \\
v_{i+1}=a_{v} t_{i+1}^{2}+b_{v} t_{i+1}+c_{v}
\end{array}\right.
$$

Where $v_{i}$ and $t$ is the wind speed and the time value at time point $i$, respectively.

Finally, the conductor temperature during the time point $i-1$ to $i+1$ can be calculated by the fourth-order Runge-Kutta arithmetic again with the small time steps. Thus, the maximum conductor temperature can be calculated and the calculated accuracy can be improved.

\section{B. Validation of Proposed Method}

To validate the proposed method, this method is used to calculate the example shown in the Section 3. Table II show the parameters of the improved model's quadratic interpolation function. Figure VII and Figure VIII show the calculated conductor temperature and the calculated error, respectively. It can be found that the maximum conductor temperature calculated with the proposed method under the discrete step of $20 \mathrm{~min}$ is $72.05^{\circ} \mathrm{C}$, which is very close to the real maximum temperature. This demonstrates that the proposed method can improve the calculated accuracy of conductor temperature model with the large discrete step.

TABLE II. THE THERMAL PARAMETERS OF THE CONDUCTOR

\begin{tabular}{|c|c|c|c|}
\hline Parameter & $\boldsymbol{a}$ & $\boldsymbol{b}$ & $\boldsymbol{c}$ \\
\hline$V$ & -0.06 & 0.1181 & 20 \\
\hline$S$ & $1.278 \times 10^{-5}$ & -0.001774 & 0.6987 \\
\hline$I$ & -0.02914 & 4.847 & 696.6 \\
\hline & & \\
\hline
\end{tabular}

FIGURE VI. THE LINE TEMPERATURE OF THE IMPROVED MODEL 


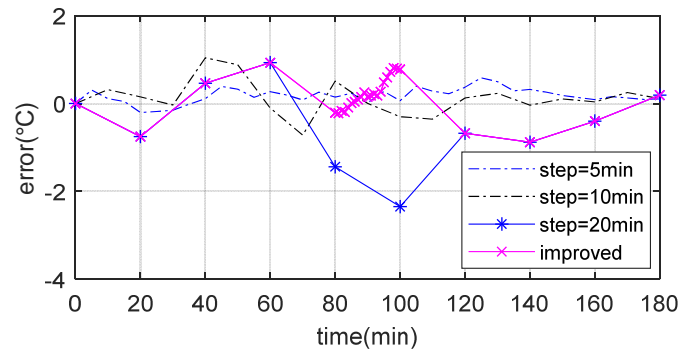

FIGURE VII. THE TEMPERATURE ERROR OF THE IMPROVED MODEL

\section{CONCLUSION}

The conductor temperature calculated with the fourth-order Runge-Kutta algorithm would be unreliable under the discrete steps, although the fourth-order Runge-Kutta algorithm have higher accuracy than the forward Euler algorithm. To improve the accuracy of overhead conductor temperature model, a method combining the fourth-order Runge-Kutta algorithm and quadratic function interpolation, is proposed in this paper. The calculating example indicates that the proposed method can improve calculated accuracy even though the model is discretized with the large discrete steps.

\section{ACKNOWLEDGMENT}

This work is supported by the "NARI Group Corporation2017 Open Fund Project from The National Key Laboratory of Smart Grid Protection and Operation Control" (Grant No. SGNR0000GZJS1705881) and "National Natural Science Foundation of China” (Grant No. 51607091).

\section{REFERENCES}

[1] Hansheng Liu, Jian Liu, Jun'e Li, Hanguang Wang, Li Guo, et al, "Conductors selection of UHVDC transmission lines based on life cycle cost,” High Voltage Engineering, Vol.38, No.2, pp.310-315, 2012.

[2] Yongchang Fu, Tao Chen, Wenbin Zhang, Zhilian Wei, Jiangang Tang, et al, "Experimental study on temperature measurement of underground cable by fiber bragg grating," Power System Technology, No.5, pp.212215, 2010.

[3] Gang Liu, Banyi Ruan, Ming Zhang, "A transient model for overhead transmission line dynamic rating based on thermal circuit method," Automation of Electric Power Systems, Vol.36, No.16, pp.58-62, 2012.

[4] Kongsen Wang, Gehao Sheng, Kui Wang, Yadong Liu, Ronghua Cui, et al, "Operation risk assessment of a transmission line dynamic line rating system,” Automation of Electric Power Systems, Vol.35, No.23, pp.1115, 2011.

[5] Bei’er Yuan, Zhanfeng Ying, Baojun Qi, Xudong Zhang and Kai Feng, "Overheating risk assessment method for high voltage transmission line using aluminum conductor composite core," Automation of Electric Power System, Vol.42, No.1, pp.111-117, 2018.

[6] ZhanFeng Ying, Yuanshen Chen and Kai Feng, "New DTR Estimation Method Without Measured Solar and Wind Data,” Journal of Electrical Engineering \& Technology, Vol.12, No.2, pp.576-585, 2017.

[7] Xiliang Ding, Xueshan Han, Hui Zhang, Yanling Wang and Kang Chang, "Analysis on electrothermal coordination power flow and transmission line temperature variation process," Proceedings of the CSEE, Vol.28, No.19, pp.138-144, 2008.

[8] IEEE Standard 738-2012, "IEEE Standard for Calculating the CurrentTemperature Relationship of Bare Overhead Conductors”.
[9] Engelhardt, J. S., and S. P. Basu. "Design, installation, and field experience with an overhead transmission dynamic line rating system," Transmission and Distribution Conference, 1996. Proceedings., IEEE, 1996, pp.366-370.

[10] Xinbo Huang, qindong Sun and Guanjun Zhang, "An on-line monitoring system of temperature of conductors and fittings in transmission lines," Electrotechnical Application, Vol.27, No.16, pp.63-67, 2008. 